SCHWERPUNKT

\section{Pervasive Computing in der medizinischen Versorgung}

\section{Einführung in den Schwerpunkt}

von Carsten Orwat, ITAS, Asarnusch Rashid,
FZI, Michaela Wölk, IZT, Carsten Holtmann, FZI,
Mandy Scheermesser und Hannah Kosow, IZT

\section{Einleitung}

\subsection{Zum Konzept des Pervasive Computing}

„Pervasive Computing” oder verwandte Konzepte der Informations- und Kommunikationstechnologien (IKT) halten vermehrt Einzug in den Gesundheitsbereich. Dabei existiert aufgrund der relativ frühen Entwicklungs- und Anwendungsphase von Pervasive Computing keine allgemeingültige Definition (Hansen et al. 2006), die zudem Pervasive Computing von verwandten Konzepten wie das „Ubiquitous Computing“, „Ambient Intelligence“, „Embedded Computing“ oder „Disappearing Computing " exakt abgrenzen würde. ${ }^{2}$ Stattdessen verwenden Autoren in der einschlägigen Literatur den Begriff Pervasive Computing mehr oder weniger stringent für die weitere Verbreitung von miniaturisierter, mobiler oder eingebetteter IKT, die einen gewissen Grad an „Intelligenz“, in den meisten Fällen Netzwerkverbindung sowie fortschrittliche Nutzerschnittstellen aufweisen (Satyanarayanan 2001; Lyytinen, Yoo 2002; Saha, Mukherjee 2003; Alcañiz, Rey 2005; Kenny 2006).

Im Folgenden wird Pervasive Computing als Leitbild der Entwicklung von IKT-Systemen verstanden, die ,ubiquitär“ in dem Sinne sind, als ihre Nutzung nicht an einen speziellen, dafür vorgesehenen Ort gebunden ist, wie z. B. an einen Computerarbeitsplatz. Dadurch lässt sich Pervasive Computing gegenüber vielen telemedizinischen Anwendungen abgrenzen, die nur eine lokal eingeschränkte Nutzung zulassen (wie z. B. bei telemedizinischen Videokonferenzen). Die „Ubiquität“ wird dabei in der Regel entweder dadurch erreicht, dass IKT in den den Nutzer umgebenden Gegenständen, Gebäuden oder in die sonstige Infrastruktur eingebettet wird, oder dadurch, dass mobile Geräte mit dem Nutzer „mitwandern“ (vgl. z. B. Banavar, Bernstein 2002). Zu den mobilen Geräten können

a) mobile Computer (z. B. Laptops, Personal Digital Assistants oder Tablet PCs) einschließlich fortschrittlicher Mobiltelefone (,Smartphones“),

b) sogenannte „Wearables“ wie Textilien, Accessoires oder medizinische Geräte mit Rechner- und Kommunikationsleistungen (Lukowicz et al. 2004; Fleisch, Michahelles 2007) sowie

c) computerisierte Implantate

gezählt werden. Des Weiteren weisen Pervasive Computing Systeme in der Regel eine spezifische Form der „Intelligenz“ auf, indem sie den Kontext der Anwendung erkennen und darauf reagieren (,context awareness") (Loke 2006; Bricon-Souf, Newman 2007; Kunze et al. 2008) oder Leistungen der Entscheidungsunterstützung bieten. Dadurch grenzen sich Pervasive-Computing-Systeme gegenüber Systemen $\mathrm{ab}$, bei denen Daten lediglich übertragen werden und auf die Verarbeitung des Kontextes verzichtet wird. Ferner erbringen PervasiveComputing-Systeme in der Regel ihre Hauptleistungen der Datenverarbeitung und -übertragung automatisiert, ohne menschliche Akteure zwingend einzubeziehen.

\subsection{Funktionen und Potenziale des Pervasive Computing in der medizinischen Versorgung}

Durch die ubiquitäre Bereitstellung der analytischen, diagnostischen, informierenden oder dokumentierenden Funktionen wird Pervasive Computing als geeignet eingeschätzt, Leistungen der medizinischen Versorgung und Pflege zu verbessern (Bott et al. 2005; Mattila et al. 2007; Wan, Taveras 2007). Beispielsweise können mit den Fähigkeiten der automatisierten Fern- und Selbstüberwachung sowie -diagnose für Patienten die Möglichkeiten der häuslichen Pflege und medizinischen Versorgung verbes- 
sert werden, ebenso aber auch die der Selbstversorgung oder der unabhängigen Lebensführung im Alter (z. B. Korhonen et al. 2003).

Ferner kann die Effizienz und Effektivität der medizinischen Leistungserbringer mit der automatisierten Dokumentation von Aktivitäten, der automatisierten Steuerung von Arbeitsprozessen sowie durch die adäquate Informationsversorgung in spezifischen Arbeitssituationen erhöht werden. Beispielsweise wird mit dem RFID-Einsatz auf Medikamenten versucht, Medikamentierungsfehler zu vermindern (Bonnabry 2005). In diesem Zusammenhang kann auch erwartet werden, dass Pervasive Computing zum Abbau der redundanten Datenerfassung sowohl in (teils mehreren) elektronischen als auch $\mathrm{Pa}$ pierversionen beitragen kann (Haux 2006).

Des Weiteren wird dem Einsatz von Pervasive-Computing-Systemen in Krankenhäusern ein hohes Nutzenpotenzial zugeschrieben, da sie mit den oben genannten Merkmalen die besonderen Arbeitsbedingungen des Krankenhauspersonals unterstützen können: $\mathrm{Zu}$ diesen Arbeitsbedingungen gehören insbesondere hohe Mobilität und ausgeprägte Kooperation bei der Leistungserbringung, die Nutzung verschiedenster und räumlich verteilter Geräte sowie ein ständiges Wechseln zwischen verschiedenen Aktivitäten (Bardram et al. 2007). Mit der Einführung von Pervasive-Computing-Systemen wird nicht zuletzt eine Veränderung der Art der medizinischen Leistungserbringung im Sinne des ,anywhere and anytime" erwartet, wobei die Anwendung im Gesundheitswesen zunehmend mit dem Begriff „Pervasive Healthcare“ bezeichnet wird (Varshney 2003; Korhonen, Bardram 2004; Muras et al. 2006).

Einen Einblick in den aktuellen Stand der Forschung und Entwicklung sowie möglicher Anwendungen von Pervasive-ComputingSystemen in der Medizin und Pflege liefern auch die beiden Veranstaltungsberichte zur „Pervasive Health“-Konferenz (Tampere, Finnland, 2008) und zum „Ambient Assisted Living"-Kongress (Berlin, 2008) in der Rubrik „Tagungsberichte“ dieses Heftes.

\subsection{Erforschung und Diskussion von Technikfolgen}

Neben den diversen Anwendungsgebieten wurden bereits früh die sozialen, ökonomi- schen, ökologischen oder ethischen Implikationen von Pervasive Computing diskutiert (Stone 2003; Bohn et al. 2004; Bohn et al. 2005; Brey 2005). Mittlerweile liegt dazu auch eine Reihe von $T A$ - $b z w$. TA-ähnlichen Projekten vor, wie beispielsweise

- das Projekt der TA Swiss zu den Umweltund Gesundheitsfolgen (Hilty et al. 2003; Hilty et al. 2004) bzw.

- zu den allgemeinen gesellschaftlichen Auswirkungen (Kenny 2006),

- Teile das Stuttgarter NEXUS-Projekts (Heesen et al. 2005),

- das BMBF-Projekt TAUCIS (Bizer et al. 2006),

- die BSI-Studie PerCEntA zu Sicherheitsund Datenschutzaspekten (Gabriel et al. 2006),

- das EU-Projekt SWAMI (Friedewald et al. 2007; Wright et al. 2008) ${ }^{1}$ oder

- der Schweizer Risikodialog (Meier, Stiftung Risiko-Dialog 2006).

Vereinzelt und ansatzweise werden die potenziellen Nutzen und Nebenwirkungen von Pervasive Computing auch speziell im Gesundheitswesen aufgezeigt. In diesem Kontext ist das im Folgenden beschriebene Projekt PerCoMed zu sehen.

\section{Das PerCoMed-Projekt}

Mit dem Projekt „Pervasive Computing in der vernetzten medizinischen Versorgung" (PerCoMed) sollten insbesondere die Chancen und Risiken von Pervasive Computing im Gesundheitswesen sowie entsprechende Treiber und Barrieren für Innovation und Diffusion untersucht werden. $\mathrm{Zu}$ den wichtigsten Elementen des Projekts zählten die Fallstudien „Stroke Angel“ und „MS Nurses“, Fokusgruppengespräche, Benutzerakzeptanzanalysen, die Analyse der internationalen Literatur, Stakeholderund Trendanalysen sowie die Analyse von Finanzierungsformen im deutschen Gesundheitswesen. Ferner diente im Projekt ein Expertenworkshop (siehe PerCoMed Projekt 2007) sowie ein Anwendertag auf der Messe „Medica“ (siehe PerCoMed Projekt 2008) zur Gewinnung und zum Austausch von Praxiserfahrungen sowie zur Vermittlung von Projektergebnissen. Über- 
greifende Kernergebnisse des Projektes werden in diesem Schwerpunktheft zusammengeführt für detaillierte Darstellungen sei auf die angegebene Literatur der Partner auf der Projektwebseite (http://www.percomed.de/) verwiesen, auf der auch die Ansprechpartner für die direkte Kontaktaufnahme aufgeführt sind.

Das Projekt wurde von Dezember 2005 bis Februar 2008 im Bereich „Innovations- und Technikanalyse" (ITA) vom Bundesministerium für Bildung und Forschung finanziell unterstützt (BMBF-Förderkennzeichen 16I1546). Zu den Projektpartnern zählten die nachfolgenden Forschungseinrichtungen:

- Institut für Zukunftsstudien und Technologiebewertung (IZT), Berlin

- FZI Forschungszentrum Informatik, Karlsruhe

- Institut für Technikfolgenabschätzung und Systemanalyse (ITAS), Forschungszentrum Karlsruhe

Ferner waren die Praxispartner, die Neurologische Klinik Bad Neustadt a. d. Saale der RhönKlinikum AG und die n-tier construct $\mathrm{GmbH}$, dem Projekt assoziiert. An der Neurologischen Klinik wurden die beiden Fallstudien durchgeführt. Beide Partner standen auch für Fachgespräche zur Verfügung.

\section{Die Artikel des Schwerpunktheftes}

Der erste Beitrag „,Der Umgang mit Barrieren bei der Einführung von Pervasive Computing. Ein Literaturüberblick" im Schwerpunkt gibt in verkürzter und übersetzter Form die Ergebnisse einer systematischen Analyse der internationalen Literatur zu Pervasive-ComputingSystemen in der Gesundheitsversorgung wieder (Orwat et al. 2008). Neben organisatorischen und datenschutzrechtlichen Problemen, die in der Literatur gefunden wurden, kann der Leser in der Darstellung von Gräfe, Orwat und Faulwasser einen Überblick über die Einsatzfelder von Pervasive Computing Systemen erhalten. Dabei lassen sich die am häufigsten anzutreffenden Systeme in fünf Typen von Pervasive Computing Systemen unterteilen:

1. Monitoring von Patienten im Heimbereich und unterwegs (z. B. mittels ubiquitärer IKT-Infrastrukturen in „Smart Homes“ oder mit Hilfe von „Wearables“ wie mobilen, Alarm gebenden Blutdruckmessgeräten),

2. Monitoring von Patienten im stationären Bereich (z. B. Erfassung und Auswertung von Vitalparametern mittels mobiler Geräte während oder nach Operationen),

3. Monitoring in Pflegeeinrichtungen (z. B. durch verteilte Sensoren oder Bilderkennung zur Erkennung und Alarmierung bei Stürzen),

4. ubiquitäre Informationssysteme für das Personal in medizinischen Einrichtungen (z. B. Tablet-PCs oder Personal Digital Assistants für Rettungskräfte, Ärzte oder Krankenschwestern zur Datenverwaltung auf den Stationen) oder

5. ubiquitäre Informationssysteme für die Logistik (z. B. RFID-Systeme für Medikamente, Blutkonserven oder Krankenhausbetten).

Die Literaturanalyse zeigte auch, dass die meisten Systeme in der Experimental- oder Prototypstufe beschrieben wurden, was zu Schlussfolgerungen führte, dass Barrieren für die Implementierung von Pervasive-Computing-Systemen in den medizinischen Regelbetrieb bestehen. Derartige Barrieren standen im Fokus des Projekts PerCoMed, insbesondere wurden diese in den beiden Fallstudien untersucht.

In den Fallstudien „Stroke Angel“" und „MS Nurses", die Anwendungsfälle aus den obigen Kategorien (1), (2) und (4) umfassen, wurden reale Anwendungsfälle mittels geeigneter Technologien ausgestattet, die Anwendung selbst wissenschaftlich analysiert und die Akzeptanz der Lösungen aufseiten einer Vielzahl von Stakeholdern (u. a. mittels Fokusgruppen und Benutzerakzeptanzanalyse) untersucht. Ein wesentlicher Schwerpunkt lag auf der Identifikation der wichtigsten „Stakeholder" sowie spezifischer Interessenkonflikte und Risiken im Themenfeld. Es wurde untersucht, inwieweit die verschiedenen Stakeholder die Einführung und Umsetzung von pervasiven Technologien in der medizinischen Versorgung fördern und / oder hemmen können. Insbesondere mit der Beobachtung praktischer Einführungsprozesse und der täglichen Nutzung der Technologien in den Fallstudien wurde die Bewertung von ursprünglich aufgestellten Thesen im Sinne einer Generalisierung der getroffenen Aussagen versucht. Dabei haben sich die Thesen auf die Implementierungen des Pervasive Computing in der medizini- 
schen Versorgung und deren Folgen bezogen. Es stellte sich bei den Fallstudien als besonders wertvoll heraus, dass in ihnen nicht nur die direkten Projekt- und Praxispartner einbezogen wurden, sondern auch weitere Einrichtungen und Unternehmen, die die Forschungsarbeit begleiteten oder praktisch unterstützten. $\mathrm{Zu}$ diesen Einrichtungen gehörten das Bayerische Rote Kreuz, Bad Neustadt a.d. Saale, die Stiftung Deutsche Schlaganfall-Hilfe, Gütersloh, die Philips Research, Aachen, und die Neurologie der Heinrich-Heine-Universität, Düsseldorf. Unter anderem war es dadurch möglich, Erkenntnisse der Fallstudien im Kontext der betroffenen Sektoren zu beurteilen. Teilweise wurden mit diesen Einrichtungen Ideen, Konzepte und Lösungsszenarien, aber auch Risiken und Hindernisse gemeinsam diskutiert, evaluiert und in wissenschaftlichen Beiträgen publiziert.

Im Rahmen der PerCoMed-Fallstudie „Stroke Angel“" wurde der Einsatz von Pervasive-Computing-Technologien zur Verbesserung des Informationsmanagements an der präklinisch-klinischen Schnittstelle in der Schlaganfallversorgung evaluiert. Im Beitrag ,,Sektorenübergreifendes Informationsmanagement im Gesundheitswesen - Neue Wege durch Pervasive Computing " von Holtmann, Rashid, Müller-Gorchs, Ziegler, Griewing und Kögerl werden die Ergebnisse vorgestellt und die Bedeutung von Pervasive Computing für ein sektorenübergreifendes Informationsmanagement diskutiert. Dabei wird vor allem auf das Problem schwer integrierbarer Leistungsketten eingegangen. Während in anderen Branchen die Leistungs- bzw. Prozessoptimierungen über die gesamte Wertschöpfungskette ein viel beachtetes Thema sind, scheitern - so die Autoren integrierte Betrachtungen im Gesundheitswesen häufig noch an institutionellen, regulatorischen und mithin auch regionalen Grenzen. Die Fallstudie „Stroke Angel“ zeigte unter anderem, dass sektorenübergreifende Lösungen zwar ein hohes medizinisches Potenzial haben können, dass die herrschenden Rahmenbedingungen das Zustandekommen derartiger Lösungen aber sowohl für die Technologieanbieter als auch die Nutzer nicht erleichtern. Obwohl gezeigt werden konnte, dass die bessere Verfügbarkeit entscheidungsrelevanter Daten durch den Einsatz pervasiver Technologien zu einer qualitativen Verbesserung der medizini- schen Versorgung führt, legt der Beitrag jedoch auch dar, dass die Schaffung von Informationstransparenz in ihren unterschiedlichsten Facetten und über die verschiedenen Stufen der Wertschöpfungskette noch eine wesentliche Forderung an Politik, Technologielieferanten, Gesundheitsdienstleister und Patienten ist.

In der zweiten Fallstudie „MS Nurses“ wurde untersucht, inwieweit Pervasive Computing bei der Behandlung und Therapie von Patienten mit Multipler Sklerose unterstützend wirken kann. Hierfür wurde eine medizinische Studie initiiert, bei der ein mit Sensoren ausgestatteter Gürtel, der actibelt ${ }^{\circledR}$, erprobt wird. Diese Studie ist gegenwärtig noch nicht abgeschlossen. Mit seiner Hilfe sollen durch eine kontinuierliche, alltagstaugliche Bewegungsmessung Gehstörungen und Aktivitätsbeeinträchtigungen frühzeitig erkannt werden, um langfristig die Therapie zu verbessern. In einem interdisziplinären Ansatz wurde der klinische Einsatz der actibelt-Technologie im Hinblick auf medizinische, soziotechnische und ökonomische Aspekte evaluiert. Die Ergebnisse dieser Studie werden in dem Beitrag „Mobiles Aktivitätsmonitoring für ambulante Dienstleistungen im Gesundheitswesen. Analyse auf Basis der klinischen Fallstudie ,MS Nurses' " von Rashid und Holtmann vorgestellt. Die Ergebnisse sollen auch als Ausgangsbasis für den breiten Einsatz innovativer Technologien in der medizinischen Aktivitätsanalyse dienen und dazu beitragen, neue Erkenntnisse über die Anwendung des Pervasive Computing in der Medizin zu gewinnen.

Technologien des Pervasive Computing können sich nur dann etablieren, wenn sie auf Zustimmung und Nachfrage stoßen. Dies gilt sowohl im Hinblick auf die unmittelbaren Nutzer der Technologien als auch für das ökonomische und gesellschaftliche Umfeld. Nicht selten werden hohe Beträge fehlinvestiert, weil sich die Defizite neuer Anwendungen nach deren Einführung als substanziell erweisen. Vor diesem Hintergrund wurden im PerCoMed-Projekt bereits frühzeitig mit Benutzerakzeptanzanalysen, Fokusgruppen, Interviews sowie Workshops möglichst viele unmittelbar und mittelbar an den neuen Anwendungen beteiligte Akteure sowie ergänzend Experten der unterschiedlichsten wissenschaftlichen Disziplinen einbezogen. Durch dieses Vorgehen konnten im Projekt si- 
cher nicht alle identifizierten Kontroversen aufgelöst, dafür aber transparent gemacht werden. Zudem konnten durch die Befragungen signifikante Verbesserungsvorschläge der erprobten Technologien sowie Vorschläge für die weitere Technikentwicklung erarbeitet werden. Der Beitrag „Pervasive Computing als Zukunftsmodell? Chancen und Risiken aus Sicht von Ärzten und Patienten " von Wölk, Scheermesser und Kosow fasst die wesentlichen Erkenntnisse der Akzeptanzuntersuchungen zu den beiden Fallstudien „Stroke Angel“ und „MS Nurses“ zusammen, greift die Frage nach den Gestaltungsbedingungen auf und generalisiert, soweit möglich, die Ergebnisse.

Im Verlauf des Projekts wurde deutlich, dass Forschungs- und Entwicklungsprojekte zu Systemen des Pervasive Computing für medizinische Anwendungen oft nicht über den Experimental- oder Prototypstatus hinauskommen. In vielen Fällen wurden dafür fehlende Finanzierungsmöglichkeiten für die anschlieBende Implementierung und Fortführung im Regelbetrieb verantwortlich gemacht. Daher wurde eingehend der regulative Rahmen des deutschen Gesundheitswesens nach Finanzierungs- und Kostenerstattungsmöglichkeiten für Pervasive-Computing-Systeme in der gesetzlichen Krankenversicherung untersucht und dabei möglichen Innovations- und Diffusionsbarrieren nachgegangen. Der Artikel „Finanzierungsfragen des Pervasive Computing im Gesundheitswesen " von Orwat und Panova gibt die Ergebnisse dieser Untersuchung wieder. Dabei können die besonderen Eigenschaften von Pervasive-Computing-Systemen die ohnehin bestehenden Innovationsbarrieren durch das Finanzierungssystem noch verstärken.

Der Beitrag ,, Weit reichende Folgen für Patienten und Ärzte - Zur Einführung der Elektronischen Gesundheitskarte" von Stachwitz beleuchtet ein zentrales Projekt der Bundesregierung im Bereich der Gesundheitstelematik: die elektronische Gesundheitskarte (eGK). Die eGK kann zum einen als ein Infrastrukturbaustein für Pervasive-Computing-Anwendungen angesehen werden, denn die Verarbeitung elektronisch erfasster Patientendaten ist bei einer Reihe von Pervasive-Computing-Systemen vorgesehen. Zum anderen ist sie auch ein Beispiel für die sektorübergreifende Durchdringung des Gesundheitswesens mit Informations- und
Kommunikationstechnologien. Obwohl ihre Entwicklung und Einführung bereits seit 2002 forciert wird, kommt die eGK nicht so recht voran; ihre Einführung ist aufgrund zahlreicher technischer und organisatorischer Probleme in den Feldtests immer wieder verschoben worden. So gab es nach Angaben der "gematik“, der Gesellschaft für Telematikanwendungen der Gesundheitskarte, beispielsweise Probleme mit dem Zugriff auf die Karten sowie mit dem Einsatz des neuen elektronischen Rezeptes (eRezept), das als eine der Hauptanwendungen der eGK beworben wird. Eine flächendeckende Versorgung wird daher nunmehr frühestens Ende 2008 bis 2009 erwartet. Der Beitrag zeigt eine durchaus skeptische Sicht auf das technologische Groß- und Referenzprojekt der Bundesregierung. So werden $u$. a. eine ungleiche Verteilung von Kosten und Nutzen des Projekts sowie fehlende wissenschaftliche Belege zum medizinischen Nutzen der eGK kritisiert, woraus der Autor den Bedarf nach Technikfolgenabschätzung ableitet.

Bereits bei der frühen Entwicklung des Ubiquitous-Computing- bzw. Pervasive-Computing-Konzepts wurde auf die potenzielle Gefährdung des Schutzes privater Daten hingewiesen (z. B. Weiser 1991, S. 75). Seitdem werden Datenschutzfragen parallel zur technischen Forschung und Entwicklung thematisiert (siehe insbesondere die unter 1.3 aufgeführten Studien und nach Lösungen gesucht, die sowohl technische und organisatorische Konzepte als auch rechtliche Ansätze umfassen. Im Beitrag ,Datenschutz bei Pervasive Computing im Gesundheitswesen" geht $\check{C}$ as auf die besondere Sensibilität des Schutzes persönlicher Daten im Gesundheitsbereich ein. Um die Gefährdungspotenziale des Pervasive Computing abzuschätzen, vergleicht er systematisch die potenziellen Fähigkeiten des Pervasive Computing mit den Prinzipien des Datenschutzes, die insbesondere mit der OECD-Richtlinie zum Schutz der Privatsphäre (OECD 1980) etabliert wurden und ihren Niederschlag im europäischen und deutschen Datenschutzrecht fanden.

Nicht nur der Schutz der persönlichen Daten ist ein ethisches Problem. Der Beitrag ,Ethische Fragen des Pervasive Computing im Gesundheitswesen" diskutiert die Bereiche „Sicherheit", „Autonomie“ und „Gerechtigkeit", die aus der Sicht von Autor Siep zu den wich- 
tigsten zählen, in denen ethische Abwägungen notwendig sind. Einerseits könne die Sicherheit in der medizinischen Versorgung mit PervasiveComputing-Systemen verbessert werden, weil z. B. Informationsfehler technisch reduziert werden können. Andererseits könnten neue technische Defekte, Bedienungsfehler und ein $\mathrm{zu}$ großes $\mathrm{Ma} ß$ an präventiven Einstellungen auftreten. Im Bereich Autonomie stehe die mögliche Verbesserung der autonomen Lebensführung bei Krankheit und im Alter einer Gefährdung der informationellen Selbstbestimmung gegenüber. Im letzten Bereich Gerechtigkeit muss - so Siep - zwischen den Möglichkeiten der Leistungssteigerung und des „Enhancement“ auf der einen Seite und der Gefährdungen der Chancen- und Verteilungsgerechtigkeit auf der anderen abgewogen werden.

\section{Schlussbetrachtung}

Rückblickend kann festgehalten werden, dass Pervasive-Computing-Systeme erhebliche Potenziale für die Verbesserung der medizinischen Versorgung haben können, insbesondere vor dem Hintergrund der zunehmenden Bedeutung von Prävention und Selbstversorgung und Forderungen nach Effizienzsteigerungen in medizinischen Einrichtungen. Dabei zeigt sich derzeit, dass zwar vielfältige Anwendungsmöglichkeiten bestehen, dass allerdings die tatsächliche Einführung in die Regelversorgung eher schleppend verläuft.

Mit der Analyse der Literatur und insbesondere mit den beiden Fallstudien sollte der sehr abstrakte Charakter des PervasiveComputing-Konzepts in der medizinischen Versorgung veranschaulicht und für detaillierte Betrachtungen zugänglich gemacht werden. Das Hauptziel des Projekts war es, die Chancen und Risiken der Einführung von PervasiveComputing-Systemen in der medizinischen Versorgung sowie die Bedingungen von Innovation und Diffusion zu untersuchen. Dabei erwies sich insbesondere das interdisziplinäre Vorgehen im Projekt als hilfreich, die Innovationsbedingungen im komplexen Netz der betroffenen Akteure zu verdeutlichen. Sie werden vor allem in der Akzeptanz durch das medizinische Personal, der adäquaten Einbeziehung der betroffenen Akteure sowie bei Fragen der Kostenerstattung gesehen.

Nicht zuletzt taucht das Finanzierungsproblem auch bei der elektronischen Gesundheitskarte auf, die als Basisinfrastruktur für IKT-Anwendungen einschließlich PervasiveComputing-Systemen in der Medizin angesehen werden kann. Ferner sind bei neuen Anwendungen des Pervasive Computing die Fragen des Datenschutzes bei Patienten und Personal sowie weitere ethische Fragen zu Sicherheit, Autonomie oder Chancengleichheit noch weitgehend ungeklärt und erfordern somit weitere Betrachtungen.

\section{Anmerkungen}

1) Wir danken der Neurologischen Klinik Bad Neustadt a. d. Saale der Rhön-Klinikum AG und der n-tier construct $\mathrm{GmbH}$ an dieser Stelle nochmals ausdrücklich für die Fachgespräche.

2) Zur Geschichte der Begriffe und zu Abgrenzungsversuchen siehe beispielsweise Lyytinen und Yoo (2002) oder Wright et al. (2008).

3) $\mathrm{Zu}$ SWAMI siehe auch den Beitrag von Friedwald und Lindner in diesem Heft in der Rubrik „Ergebnisse aus TA-Projekten“

\section{Literatur}

Alcañiz, M.; Rey, B., 2005: New Technologies for Ambient Intelligence. In: Riva, G. et al. (Hg.): Ambient Intelligence. The Evolution of Technology, Communication and Cognition towards the Future of Human-Computer Interaction. Emerging Communication: Studies on New Technologies and Practices in Communication 6. Amsterdam, S. 3-15

Banavar, G.; Bernstein, A., 2002: Software infrastructure and design challenges for ubiquitous computing applications. In: Communications of the ACM 45/12 (2002), S. 92-96

Bardram, J.E.; Baldus, H.; Favela, J., 2007: Pervasive computing in hospitals. In: Bardram, J.E.; Mihailidis, A.; Wan, D. (Hg.): Pervasive Computing in Healthcare. Boca Raton, FL, S. 49-77

Bizer, J.; Dingel, K.; Fabian, B. et al., 2006: Technikfolgenabschätzung Ubiquitäres Computing und Informationelle Selbstbestimmung. Studie im Auftrag des Bundesministeriums für Bildung und Forschung. Kiel, Berlin: Unabhängiges Landeszentrum für Datenschutz Schleswig-Holstein, Institut für Wirtschaftsinformatik der Humboldt-Universität zu Berlin; http://www.taucis.hu-berlin.de/_download/T AUCIS_Studie.pdf (download 8.5.08) 
Bohn, J.; Coroama, V.; Langheinrich, M. et al., 2004: Living in a world of smart everyday objects Social, economic, and ethical implications. In: $\mathrm{Hu}-$ man and Ecological Risk Assessment 10/5 (2004), S. 763-785

Bohn, J.; Coroama, V.; Langheinrich, M. et al., 2005: Social, Economic, and Ethical Implications of Ambient Intelligence and Ubiquitous Computing. In: Weber, W.; Rabaey, J.; Aarts, E. (Hg.): Ambient Intelligence. Berlin, S. 5-29; http://www.vs.inf.ethz. ch/publ/papers/socialambient.pdf (download 8.5.08)

Bonnabry, P., 2005: Information technologies for the prevention of medication errors. In: Chimia 59/6 (2005), S. 359-361

Bott, O.J.; Ammenwerth, E.; Brigl, B. et al., 2005: The challenge of ubiquitous computing in health care: Technology, concepts and solutions - Findings from the IMIA Yearbook of Medical Informatics 2005. In: Methods of Information in Medicine 44/3 (2005), S. 473-479

Brey, P., 2005: Freedom and Privacy in Ambient Intelligence. In: Ethics and Information Technology 7/3 (2005), S. 157-166

Bricon-Souf, N.; Newman, C.R., 2007: Context awareness in health care: A review. In: International Journal of Medical Informatics 76/1 (2007), S. 2-12 Brown, I.; Adams, A.A., 2007: The ethical challenges of ubiquitous healthcare. In: International Review of Information Ethics 8/12 (2007), S. 53-60 Fleisch, E.; Michahelles, F., 2007: Messen und Managen - Bedeutung des Ubiquitous Computing für die Wirtschaft. In: Mattern, F. (Hg.): Die Informatisierung des Alltags. Leben in smarten Umgebungen. Berlin, S. 145-159

Friedewald, M.; Vildjiounaite, E.; Punie, Y. et al., 2007: Privacy, identity and security in ambient intelligence: A scenario analysis. In: Telematics and Informatics 24/1 (2007), S. 15-29

Gabriel, P.; Bovenschulte, M.; Hartmann, E. et al., 2006: Pervasive Computing: Entwicklungen und Auswirkungen. Bonn: Bundesamt für Sicherheit in der Informationstechnik; http://www.bsi.bund.de/ literat/studien/percenta/Percenta_bfd.pdf (download 8.5.08)

Hansen, T.R.; Bardram, J.E.; Soegaard, M., 2006: Moving Out of the Lab: Deploying Pervasive Technologies in a Hospital. In: IEEE Pervasive Computing 5/3 (2006), S. 24-31

Haux, R., 2006: Health information systems - past, present, future. In: International Journal of Medical Informatics 75/3-4 (2006), S. 268-281

Heesen, J.; Hubig, Chr.; Siemoneit, O. et al., 2005: Leben in einer vernetzten und informatisierten Welt. Context-Awareness im Schnittfeld von Mobile und Ubiquitous Computing. Nexus-Schriftenreihe, Stuttgart: Universität Stuttgart, Sonderforschungsbereich
SFB 627 Nexus; http://www.uni-stuttgart.de/philo/ index.php id $=65 \&$ no_cache $=1 \&$ file $=337 \&$ uid $=761$ (download 8.5.08)

Hilty, L.; Behrendt, S.; Binswanger, M. et al., 2003: Das Vorsorgeprinzip in der Informationsgesellschaft. Auswirkungen des Pervasive Computing auf Gesundheit und Umwelt. Studie des Zentrums für Technologiefolgen-Abschätzung, Bern: TA-SWISS; http:/www.ta-swiss.ch/a/info_perv/2003_46_ pervasivecomputing_d.pdf(download 8.5.08)

Hilty, L.M.; Som, C.; Kohler, A., 2004: Assessing the human, social, and environmental risks of pervasive computing. In: Human and Ecological Risk Assessment 10/5 (2004), S. 853-874

Kenny, L., 2006: Exploring the Business and Social Impacts of Pervasive Computing. Zurich: IBM Zurich Research Laboratory, Swiss Re Centre for Global Dialogue, TA-SWISS; Published by Swiss Reinsurance Company; http://www.ta-swiss.ch/a/info _perv/060506_DIV_Pervasive_computing_brochur e_e.pdf (download 8.5.08)

Korhonen, I.; Bardram, J.E., 2004: Guest Editorial. Introduction to the Special Section on Pervasive Healthcare. In: IEEE Transactions on Information Technology in Biomedicine 8/3 (2004), S. 229-234

Korhonen, I.; Parkka, J.; Van Gils, M., 2003: Health monitoring in the home of the future. In: IEEE Engineering in Medicine and Biology Magazine 22/3 (2003), S. 66-73

Kunze, C.; Holtmann, C.; Schmidt, A. et al., 2008: Kontextsensitive Technologien und Intelligente Sensorik für Ambient-Assisted-Living-Anwendungen. 1. Deutscher Kongress „Ambient Assisted Living“ (AAL 08), Berlin

Loke, S.W., 2006: Context-aware artifacts: two development approaches. In: IEEE Pervasive Computing 5/2 (2006), S. 48-53

Lukowicz, P.; Kirstein, T.; Troster, G., 2004: Wearable systems for health care applications. In: Methods of Information in Medicine 43/3 (2004), S. 232-238

Lyytinen, K.; Yoo, Y.J., 2002: Issues and challenges in ubiquitous computing. In: Communications of the ACM 45/12 (2002), S. 62-65

Mattila, E.; Korhonen, I.; Saranummi, N., 2007: Mobile and personal health and wellness management systems. In: Bardram, J.E.; Mihailidis, A.; Wan, D. (Hg.): Pervasive Computing in Healthcare. Boca Raton, S. 105-134

Meier, K.; Stiftung Risiko-Dialog, 2006: Stakeholder-Dialog II über Pervasive Computing. Aussichten und Einsichten. riskDOK 004. Fakten aus dem Risiko-Dialog, St. Gallen: Stiftung Risiko-Dialog; http://www.ictswitzerland.ch/files/webcontent/news /riskDOK004.pdf (download 8.5.08)

Muras, J.A.; Cahill, V.; Stokes, E.K., 2006: A Taxonomy of Pervasive Healthcare Systems. 1st Interna- 
tional Conference on Pervasive Computing Technologies for Healthcare 2006, Innsbruck, Austria, November 2006; http://ieeexplore.ieee.org/iel5/42051 39/4205140/04205171.pdf?tp=\&isnumber $=\&$ arnumb er=4205171 (download 8.5.08)

OECD - Organisation for Economic Co-operation and Development, 1980: OECD Guidelines on the Protection of Privacy and Transborder Flows of Personal Data. Paris; http://www.oecd.org/document/18/ 0,3343,en_2649_34255_1815186_1_1_1_1,00.html (download 8.5.08)

Orwat, C.; Graefe, A.; Faulwasser, T., 2008: Towards Pervasive Computing in Healh Care - A Literature Analysis. In: BMC Medical Informatics and Decision Making (eingereicht)

PerCoMed Projekt, 2007: Bericht zum PerCoMedExpertenworkshop. PerCoMed Report (Projekt PerCoMed - Pervasive Computing in der medizinischen Versorgung), Karlsruhe: ITAS im Forschungszentrum Karlsruhe; http://www.percomed.de/images/sto ries/expertenworkshop/percomed_workshopbericht_f inal.pdf (download 8.5.08)

PerCoMed Projekt, 2008: Intelligent, vernetzt, mobil, alltagstauglich - Technologien der Zukunft für die medizinische Versorgung von heute. Kurzzusammenfassung des Abschlussworkshop des Forschungsprojektes „PerCoMed - Pervasive Computing in der vernetzten medizinischen Versorgung“" Medica Media 2007, Düsseldorf, 16.11.2007. PerCoMed Report (Projekt PerCoMed - Pervasive Computing in der medizinischen Versorgung), Karlsruhe und Berlin: ITAS im Forschungszentrum Karlsruhe; Forschungszentrum Informatik; Institut für Zukunftsstudien und Technologiebewertung; http://www.percomed.de/images/stories/medica200 7/percomed_bericht_zum_medicamedia_workshop. pdf (download 8.5.08)

Rigby, M., 2006: Ubiquitous technologies in health: new challenges of opportunity, expectation, and responsibility. In: Studies in Health Technology and Informatics 124 (2006), S. 65-70

Rigby, M., 2007: Applying emergent ubiquitous technologies in health: The need to respond to new challenges of opportunity, expectation, and responsibility. In: International Journal of Medical Informatics 76/Supp. 3 (2007), S. S349-S352

Saha, D.; Mukherjee, A., 2003: Pervasive computing: a paradigm for the 21st century. In: IEEE Computer 36/3 (2003), S. 25-31

Satyanarayanan, M., 2001: Pervasive computing: vision and challenges. In: IEEE Personal Communications 8/4 (2001), S. 10-17

Stone, A., 2003: The dark side of pervasive computing. In: IEEE Pervasive Computing 2/1 (2003), S. 4-8 Varshney, U., 2003: Pervasive healthcare. In: IEEE Computer 36/12 (2003), S. 138-140
Wan, D.; Taveras, L.E., 2007: The business of pervasive healthcare. In: Bardram, J.E.; Mihailidis, A.; Wan, D. (Hg.): Pervasive Computing in Healthcare. Boca Raton, FL, S. 275-298

Weiser, M., 1991: The Computer for the 21st Century. Specialized elements of hardware and software, connected by wires, radio waves and infrared, will be so ubiquitous that no one will notice their presence. In: Scientific American (International Edition) 265/3 (1991), S. 66-75

Wright, D.; Gutwirth, S.; Friedewald, M. et al. (Hg.), 2008: Safeguards in a World of Ambient Intelligence. Heidelberg

\section{Kontakt}

Dr. Carsten Orwat

Institut für Technikfolgenabschätzung und Systemanalyse (ITAS)

Forschungszentrum Karlsruhe in der HelmholtzGemeinschaft Postfach 36 40, 76021 Karlsruhe

Tel.: +49 (0) 7247 / 82 - 6116

E-Mail: orwat@itas.fzk.de 